\title{
Socioeconomic Status and Relative Incidence of Hysterectomy and Endometrial Ablation in Young Australian Women
}

\begin{abstract}
Decisions regarding surgery are complex and economic influences affect choices made both by patients and their doctors. There is evidence that surgeons' decisions to offer operations are affected by financial incentives, yet we could find no studies addressing whether, once a decision to operate is made and a choice of procedures is available, operations offering greater financial reward are favoured. The choice between endometrial ablation or hysterectomy in heavy menstrual bleeding offers an opportunity to study decision-making. We obtained on all private hospital claims made in Australia for either endometrial ablation or hysterectomy for women aged 30 to 50 years for the five-year period 2012 to 2016 inclusive, according to socioeconomic status. The overall incidence rates and the ratio between hysterectomy and ablation, and the association between socio-economic factors, were examined using linear regression. We found that the surgery with the greatest economic impact on the patient (hysterectomy) was more commonly performed than ablation, yet hysterectomy became the less dominant choice with increasing socioeconomic status of women. This finding suggests that direct financial costs are a lesser consideration in choice of the procedure with patients, but that surgeons may respond to a financial incentive to perform a more expensive procedure.
\end{abstract}

Keywords: Surgery; Socioeconomic; Patient; Choice; Supplier; Demand

\section{Louise Rawlings ${ }^{1}$, Pauline O'Shaughnessy ${ }^{2}$, Agnes Wilson ${ }^{3}$ and Stephen J Robson ${ }^{4 *}$}

1 Crawford School of Public Policy, Australian National University, Acton, Canberra, 2600, Australia

2 School of Mathematics and Applied Statistics, Faculty of Engineering and Information Science, University of Wollongong, NSW 2500, Australia

3 Health Research Consulting, Sydney, NSW, Australia

4 Medical School, the Australian National University, Garran, Canberra, 2605, Australia

\section{*Corresponding author: Stephen J Robson \\ Đstephen.robson@anu.edu.au}

Medical School, the Australian National University, Garran, Canberra, 2605, Australia.

Tel: +61262823033

Received: June 06, 2019; Accepted: June 19, 2019; Published: June 26, 2019

\section{Introduction}

Health care is expensive, and likely to become more so in the future. An analysis of trends in health expenditure at a global level estimated that total spending is likely to increase from US\$9.21 trillion in 2014 to as much as $\$ 30$ trillion by 2040 [1]. Increases in health spending are driven by continued growth in GDP, government spending, and government health spending and affect not only high-income countries: expenditure in lowermiddle income countries predicted to grow at annual rates of more than $4 \%$ for the foreseeable future [1].

Internationally Australia's healthcare system and its outcomes rank within the top ten countries for healthcare efficiency. ${ }^{1}$ Despite this, "many healthcare professionals, policymakers, and government officials share the view that although the quality of care is high, [Australia's] healthcare spending is unsustainable and

${ }^{1}$ Bloomberg. Where do you get the most for your health care dollar? Published September 18, 2014. Accessible at: https://www.bloomberg.com/graphics/ infographics/most-efficient-health-care-around-the-world.html
Citation: Rawlings L, O'Shaughnessy P, Wilson A, Robson SJ (2019) Socioeconomic Status and Relative Incidence of Hysterectomy and Endometrial Ablation in Young Australian Women. J Health Med Econ Vol.5 No.1:2

a drain on the economy" [2]. The Australian Institute of Health and Welfare report Health Expenditure Australia 2015-2016 estimated that health spending comprised $10.3 \%$ of GDP at just over AUD\$ 170 billion, a $3.6 \%$ increase in real terms over the year before. $^{2}$ However, the yearly increase in health expenditure has averaged 4.7\% since 2005-2006. Government expenditure represents about two thirds of all health expenditure, estimated to be just under AUD\$115 billion for 2015-2016. The total expenditure of public hospitals by all levels of government was $\$ 51.1$ billion in 2015-2016. In Australia, 68\% of health care costs are funded through the public health system, with $32 \%$ from other sources,

${ }^{2}$ Australian Institute of Health and Welfare 2017. Health expenditure Australia 2015 16. Health and welfare expenditure series no. 58. Cat. no. HWE 68. Canberra: AlHW. 
including private health insurers and out-of-pocket expenses. ${ }^{3}$ To encourage Australians to take out health insurance, the private health system is subsidised by a private health insurance rebate, which costs the public about AUD\$5 billion per year.

\section{Demands in health care}

It is well-established that demand for medical care is relatively inelastic with respect to price. Standard economics tends to assume that supply and demand schedules in a given market are independent [3]. Fuchs [3] points out that medical care has two components: the nominal price charged by the doctor; and, the value of the patient's time. In addition to the cost of the treatment or service, the patient will face an additional cost from the time taken to undergo the procedure, including lost work time and costs associated with recovery.

A number of authors have questioned the effect of financial incentives on decision-making by medical doctors. As Shafrin [4] poses the question, "Are doctors perfect agents for their patients, solely basing their medical-care decisions on what is in the patient's best interest or do physicians behave as homo economicus, strictly acting in a profit maximising fashion?" The question of agency is fundamental to understanding the provision of services in health care, with a general assumption that the health care market reflects the standard agency relationship of the ill-informed 'principal' and the informed 'agent' seeking to optimise their utility functions [5]. Fuchs [3] explores the physician-patient relationship and makes the point that if a patient had full information and full control over the 'quantity' of care received, they would likely choose the same quantity (' $Q$ ') as the physician acting as a perfect agent. However, while there is consensus that the physician acts in the patient's best interests, it is not so clear exactly what constitutes 'best interests' [6]. This question - what are a patient's 'best interests?' - has such an effect on the delivery of health care that it has been considered by the Productivity Commission [7]. The Commission's report pointed out that patients were unlikely to be, "the best people to judge the impacts on their welfare of consuming different levels of care." Fuchs [8] also points out that patients commonly wish for, "any and all care that might possibly be of net benefit, regardless of cost," and physicians commonly accede to such demands either to keep the patient's goodwill or protect against malpractice litigation. Fuchs terms this, "the doctor's dilemma," as an example of demand shifting and states that a quantity of health care that maximises a patient's utility $Q$ regardless of cost is not necessarily "unnecessary care."

The Productivity Commission report, however, expresses concern that doctors may use "discretionary power" to bring about demand shifting that differs from what an "informed patient" would consider appropriate care [7]. Yet even the Commission concedes that this supplier-induced demand (SID), while considered "unwelcome and undesirable," may have benefits. For example, a doctor might persuade a patient to undertake more treatment where the patient would otherwise have opted for less effective care. Overall, though, the Productivity Commission

${ }^{3}$ Australian Institute of Health and Welfare 2016. 25 years of health expenditure in Australia 1989-90 to 2013-14. Health and welfare expenditure series no. 56. Cat. no. HWE 66. Canberra: AlHW. expresses concerns about choice of surgical and other medical treatments because these could: "result in a higher than necessary share of the nation's resources being devoted to health care and could lead to a deterioration in the health of some patients if the treatment is inappropriate [Undermining] the effectiveness of copayments and price/fee controls as a means of restraining health costs." [7].

In Australia, Medicare is intended to provide universal access to health services through the Federal Government Health Department's Medical Benefits Schedule (MBS) ${ }^{4}$ and Pharmaceutical Benefits Schedule (PBS). ${ }^{5}$ This accounts for almost one third of all Federal Government health spending: expenditure through the MBS in 2017-2018 was AUD\$23.2 billion. ${ }^{5,6}$ Approximately $17 \%$ of spending in the MBS is for surgery and procedural treatments. Thus, surgery and selection of operations has a substantial impact on government expenditure.

\section{Surgeons' decision making}

In countries not bound by 'managed care' systems - where patients and surgeons have incentives to select less costly forms of care [9]-decisions about whether operations and procedures are necessary typically are left to doctors [10]. In many cases, however, surgeons themselves may be uncertain in their decision making and there is strong evidence that clinical practice may be idiosyncratic [11-13]. In those clinical situations where surgeons disagree on the optimal procedure for their patients, rates of procedures and operations tend to vary. This phenomenon - known as 'clinical variation' - is well-recognised and a useful definition of clinical variation used in the Medical Journal of Australia is: "Patients with similar diagnoses, prognoses and demographic states receive different levels of care depending on when, where, and by whom they are treated, despite agreed and documented evidence of best practice" [13].

The Australian Commission on Safety and Quality in Healthcare (ACSQHC) released the Second Australian Atlas of Healthcare Variation in 2017, examining trends in a number of medical procedures in Australia. ${ }^{7}$ According to ACSQHC:

"Variation exists at a clinician level (between clinicians), at the service level (between different health services) and at a geographic level (between regions and countries) [and is] ubiquitous and persistent. Studies have documented its existence over decades, with some researchers indicating that it is hard to find examples where there is little or negligible variation."

The choice about whether a patient should have an operation represents "a complex interaction where multiple incentives exert competing influences on doctors, patients, and policymakers." [14] Such choice is typically a 'negotiation' between patient and doctor, and economic influences are likely to play a part

${ }^{4}$ http://www.mbsonline.gov.au/internet/mbsonline/publishing.nsf/Content/Home ${ }^{5}$ http://www.pbs.gov.au/pbs/home;jsessionid=113u6smtgljba1t0t770oaoope

${ }^{6}$ Australian Institute of Health and Welfare. 2016. 25 years of health expenditure in Australia $1989-90$ to 2013-14. Health and welfare expenditure series no. 56. Cat No. HWE 63. Canberra: AlHW. Accessible at: http://www.aihw.gov.au/publicationdetail/?id=60129554398.

${ }^{7}$ Australian Commission on Safety and Quality in Healthcare. The second Australia atlas of healthcare variation 2017. Accessible at: https://www.safetyandquality.gov.au/ atlas/atlas-2017/ 
in decision making. There are studies suggesting that 'gifts' from pharmaceutical companies are associated with more prescriptions per patient, more costly prescriptions, and a higher proportion of branded prescriptions [15]. Indeed, gifts of any size were associated with an effect and larger gifts appeared to have a greater impact on prescribing behavior.

In contrast to prescribing of medications, little research has been undertaken into the economic factors affecting either patients' or their surgeons' choices for surgery [16]. One study from the United States reported that financial incentives had little effect on surgeons' decisions to perform an operation [17]. There is also evidence from the United States that once a surgical procedure has been agreed for a patient, surgeons are more likely to perform profitable operations and procedures in ambulatory surgery centers in which they have a financial stake, rather than hospitals in which they have no equity [18]. An older study from the United States, using data from 1997-98 found that surgeons paid through fee-for-service (FFS) systems had increased rates of surgery [4]. While there is evidence that financial incentives may play a role in the decision to perform a surgical procedure, we could find no studies addressing the question of whether financial incentives have any effect on the type of operation that is performed. It would be easy to hypothesise that, all other factors being equal, surgeons might respond to an incentive and perform the operation that offers greatest financial reward.

\section{Patients' decision making}

However, while SID might operate it remains unclear the reasons why, and extent to which, patients devolve decision making about their medical care to their doctor; and, the extent to which patients engage in 'consumerist' behaviour (that is, are assertive, critical and prepared to 'shop-around'), which could be a significant constraint on doctors inducing demand [7].

When non-surgical treatments are considered, prescription of a long-term medication for example, there is a body of evidence to suggest that patients take costs into account $[19,20]$. Studies have reported that patients with diabetes may be willing to trade off the cost of long-term medications against effectiveness and side effects: one study reported that a third of patients wanted their doctors to provide information about lower cost drugs with potentially greater side effects or lower effectiveness [21].

\section{Surgical treatment of heavy menstrual bleeding as a natural experiment}

An opportunity to study the effect of financial incentives on choice of operation exists. Of the procedures with the greatest degree of variation are those for heavy menstrual bleeding $(\mathrm{HMB})$ in women. $\mathrm{HMB}$ is a major public health problem, affecting approximately $30 \%$ of premenopausal women in Australia [22]. It is associated with lower quality of life, loss of productivity, and increase healthcare expenses [23]. Surgical treatment of $\mathrm{HMB}$ commonly follows failed or ineffective medical therapy.

There are two common surgical procedures to treat $\mathrm{HMB}$ : hysterectomy (removal of the uterus) and endometrial ablation (removal only of the lining of the uterus). Hysterectomy is a major procedure, usually requiring several days in hospital and a recovery time of up to a month or more. Ablation, in contrast, is considered a minor procedure and most women are able to return to their usual activities the following day. Economic modelling has demonstrated strong financial favourability for endometrial ablation versus hysterectomy from the perspective both of the health system and the patient [24,25].

The Atlas of Clinical Variation ${ }^{8}$ references the difference in rates of hysterectomy and ablation as one of the areas of greatest clinical variation of all procedures in Australia, observing a "seven-fold difference between the lowest and highest rates of hysterectomy and a 21-fold difference in rates of endometrial ablation. This finding confirms there is marked variation in use of each procedure across Australia." In response, ACQSHC developed a clinical care standard about the treatment of $\mathrm{HMB}$ as its first piece of work flowing from Atlas' findings.

The dichotomous choice between hysterectomy and ablation provides an opportunity to study the interplay between medical recommendations and trade-offs that patients (and their families) might make. While many of these procedures are performed in public hospitals where defined treatment protocols apply, in the private health system (where the majority of elective operations are performed in Australia) there will be an opportunity to study decision-making.

The study period was ideal for economic analysis as there was no significant change in the national incidence rate of hysterectomy in women aged 35 to 54 years ${ }^{9}$, and government funding policy for the MBS has kept financial rebates for surgery unchanged. Payments made to surgeons over the study period remained fixed due to the Commonwealth Government 'Medicare freeze' policy: for endometrial ablation the range was from AUD $\$ 449.60$ to a maximum of AUD \$752.65; for hysterectomy, it varied from AUD $\$ 674.70$ to a maximum of AUD $\$ 1893.40$ depending upon the complexity of the procedure. Surgeons could potentially take into account the payment they will receive for a procedure when recommending between hysterectomy or ablation (an example of SID), and patients (and their families) would be expected to take into account not only the direct costs of a treatment but also the associated costs - time away from work and other activities. Thus, we hypothesised that patients in lower socioeconomic groups would be more likely to choose and undergo ablation, while those in higher socio-economic groups would be more likely to choose a definitive procedure as they would have a greater capacity to absorb the associated costs.

\section{Data}

Operations in public hospitals are funded through individual state and territory health departments, but surgery in private hospitals is funded in part by the Federal Government through the MBS. We obtained data on all claims made through the MBS from the Australian Department of Human Services for private operations for either endometrial ablation or hysterectomy for women aged

${ }^{8} \mathrm{https}: / / w w w . s a f e t y a n d q u a l i t y . g o v . a u / a t l a s / a t l a s-2017 /$

${ }^{9} \mathrm{https} / / / \mathrm{www}$. aihw.gov.au/reports/hospitals/procedures-data-cubes/contents/datacubes 
30 to 50 years for the five-year period 2012 to 2016, by statistical area (SA3). ${ }^{10}$

Hysterectomy data were only considered where there was no diagnosis of cancer. We also wished to confirm the assumption that most hysterectomies performed in women aged 50 years or less would be for treatment of heavy menstrual bleeding. To do this we extracted data from the Australian institute of Health and Welfare (AIHW) hospital admissions dataset ${ }^{11}$ which codes separation statistics by principal diagnosis according to the ICD-10-AM classification. During the study period 201617, admission groups coded N92 (excessive, frequent, and irregular menstruation N92.0 - N92.4) comprised 88.9\% (25011 of 28136) principal reasons for the procedure, compared to prolapse (N81.2 to N82.4, 1131 separations) and all other noninflammatory disorders of the uterus (N85, 1994 separations). At the national level, there is no data linkage showing the ICD diagnosis codes for procedures, which made it impossible to confirm the primary reason for the hysterectomy. However, there is no evidence from Australia that SES is related to diagnosis so we undertook the analysis assuming a Bayesian probability over the multiple individual statistical areas. Point estimates of the female population aged 30 to 50 years in each SA3 were obtained from the Australia Bureau of Statistics (ABS) to allow a mean age-stratified population for calculation of procedures rates per 10000 women yearly over the study period.

As our measure of socioeconomic status we used the ABS 'SocioEconomic Indexes for Areas' (SEIFA). ${ }^{12}$ SEIFA ranks statistical areas in Australia according to relative socio-economic advantage and disadvantage, and is based on information from the five-yearly Australian National Census. Each SA3 consists of a number of smaller geographical areas with different SEIFA scores, so we obtained data for the minimum and maximum SEIFA area contained within each SA3. This provided three measures of socio-economic statute for each SA3, the broad SEIFA decile classification, as well as the minimum and maximum SEIFA for each SA3. To examine the relative proportion for each of the two procedures, we calculated the ratio of hysterectomy to ablation procedure for each SA3, and then developed a scatter plot of the ratio by both minimum and maximum SEIFA for each SA3.

\section{Data Analysis}

Data were extracted to Excel ${ }^{\mathrm{TM}}$ spreadsheets and statistical analysis was performed in GenStat. ${ }^{13}$ The statistical analysis was performed on the ratio of the incidence rates between hysterectomy and ablation, and the association between socioeconomic factors and the choice of procedure was examined using linear regression. The analyses used log-transformed ratio data to satisfy the homogeneity of the variance assumption for linear regression.

${ }^{10}$ Australian Bureau of Statistics. Accessible at: www.abs.gov.au/ausstats/ abs@.nsf/Lookup/by\%20Subject/1270.0.55.001 July\%202016 Main\%20 Features Statistical\%20Area\%20Level\%203\%20(SA3) 10015

${ }^{11}$ Accessible at: https://reporting.aihw.gov.au/Reports/openRVUrl.do

${ }^{12}$ Australian Bureau of Statistics. Accessible at: http://www.abs.gov.au/websitedbs/ censushome.nsf/home/seifa

${ }^{13} \mathrm{https}: / / w w w . v s n i . c o . u k / s o f t w a r e / g e n s t a t /$

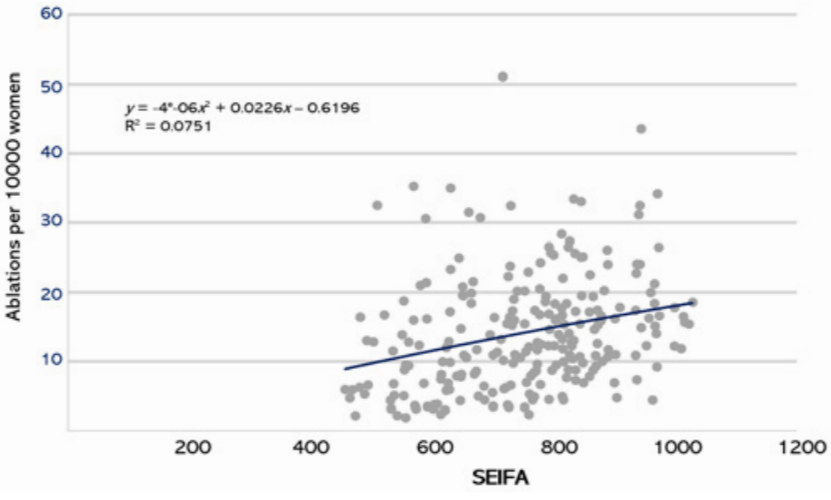

Figure 1 Age-stratified incidence rates of endometrial ablation (procedures per 10000 women) in women aged 30 to 50 years for the period 2012 to 2016 inclusive according to the lowest socio-economic area (SEIFA) in each statistical area (SA3) in Australia.

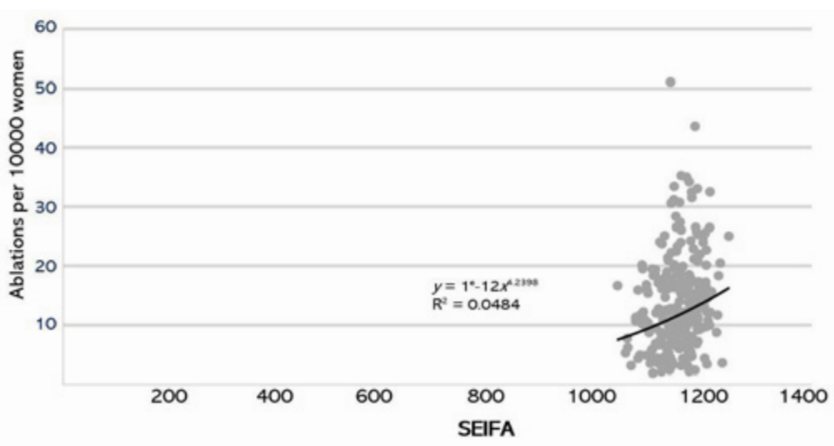

Figure 2 Age-stratified incidence rates of endometrial ablation (procedures per 10000 women) in women aged 30 to 50 years for the period 2012 to 2016 inclusive according to the highest socio-economic area (SEIFA) in each statistical area (SA3) in Australia.

\section{Results and Discussion}

In the first instance we examined the overall age-stratified incidence rates of endometrial ablation and hysterectomy for each SA3. Since each SA3 contained areas of different SEIFAs, we constructed plots of the incidence rates by the lowest and highest SEIFAs in each statistical area. Figures $\mathbf{1}$ and $\mathbf{2}$ shows scatter plots of the incidence rates (cases per 10000 women) in the lowest and highest SEIFAs in each area for endometrial ablation, and Figures $\mathbf{3}$ and $\mathbf{4}$ show the same for hysterectomy.

We examined the ratio of hysterectomy to ablation for each SA3 area, and its association with the corresponding area SEIFA decile classification. We then focused on more in-depth analyses of the relationship between the choice of treatment and the detailed SEIFA scores, and whether this relationship held across different area SEIFA deciles.

Table 1 shows the mean and the average of the ratio of incidence rates of hysterectomy to endometrial ablation for each area SEIFA decile: we observed a greater incidence of hysterectomy compared to ablation across all SEIFA deciles. The preponderance 
of hysterectomy became significantly lower in the higher the SEIFA deciles $(p<0.01)$ although the ratios were still above one, meaning an equal dichotomy between two procedures.

To further investigate the socio-economic impact on the choice of the procedure, we obtained the minimum and maximum SEIFA

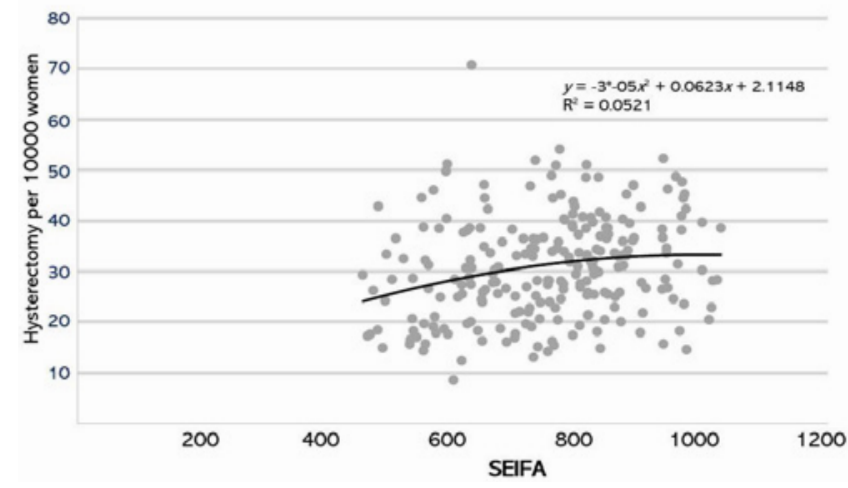

Figure 3 Age-stratified incidence rates of hysterectomy for benign indications (procedures per 10000 women) in women aged 30 to 50 years for the period 2012 to 2016 inclusive according to the lowest socio-economic area (SEIFA) in each statistical area (SA3) in Australia.

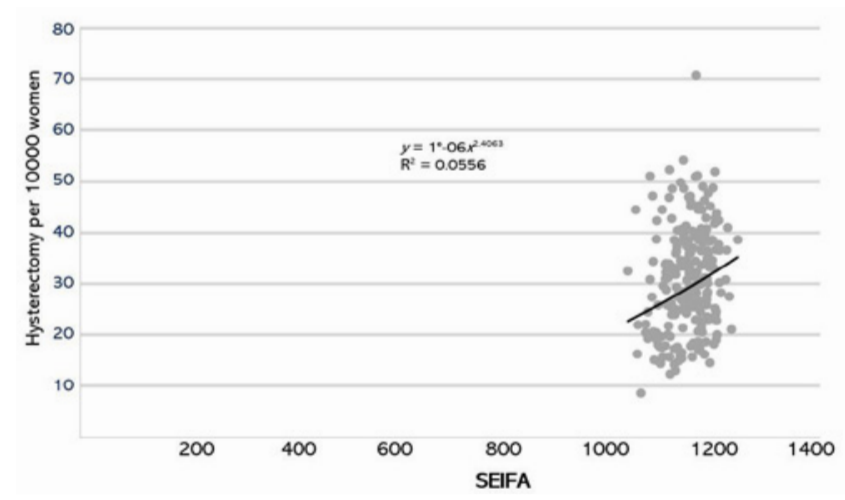

Figure 4 Age-stratified incidence rates of hysterectomy for benign indications (procedures per 10000 women) in women aged 30 to 50 years for the period 2012 to 2016 inclusive according to the highest socio-economic area (SEIFA) in each statistical area (SA3) in Australia. scores within each SA3 and the corresponding ratios of incidence rates of hysterectomy to ablation within each SA3 area. Figures 5 and 6 illustrate the association between minimum and maximum SEIFA scores and procedure of choice. The results were consistent with the previous observation - that as the SEIFA score increased, the ratio of hysterectomy to ablation decreased. Detailed

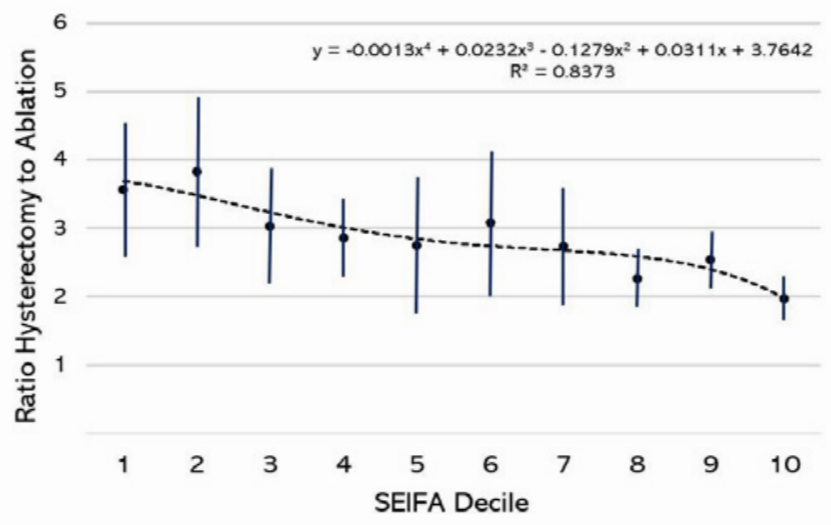

Figure 5 Ratio of hysterectomy to endometrial ablation according to the SEIFA decile for the lowest SEIFA in each SA3 $\left(a R^{2}=0.78, p<0.005\right)$.

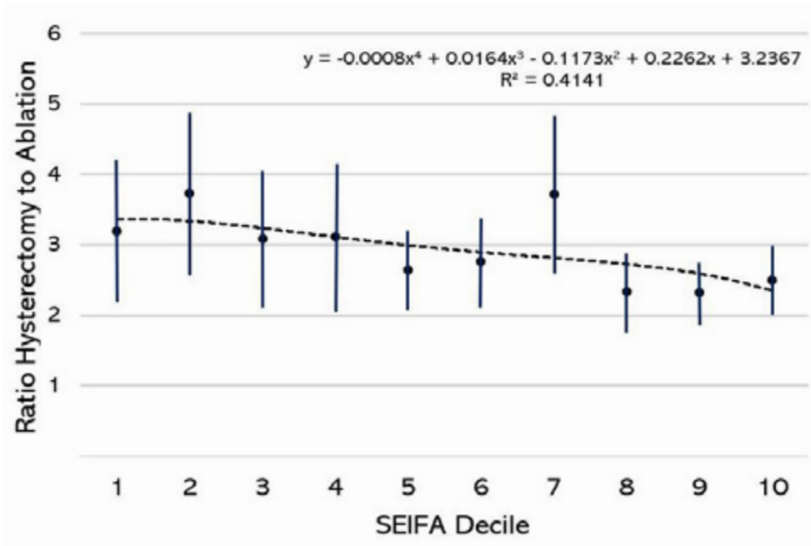

Figure 6 Ratio of hysterectomy to endometrial ablation according to the SEIFA decile for the highest SEIFA in each SA3 $\left(a R^{2}=0.33, p=0.048\right)$.

Table 1 Summary statistics for the average ratio of hysterectomy to ablation grouped by the SEIFA decile for each SA3 area and P value to test the differences between area SEIFA deciles using ANOVA.

\begin{tabular}{|l|l|l|l|l|l|l|l|l|l|l|}
\hline SEIFA Decile & 1 & 2 & 3 & 4 & 5 & 6 & 7 & 8 & 9 & 10 \\
\hline Mean & 3.57 & 4.08 & 3.52 & 2.71 & 2.88 & 2.59 & 3.04 & 2.56 & 2.07 & 2.36 \\
\hline SD & 2.91 & 1.89 & 1.45 & 2.03 & 1.29 & 1.27 & 3.06 & 2.29 & 0.59 & 0.73 \\
\hline P value & $<0.001$ (F statistic=3.462) & & & & & & &
\end{tabular}

Table 2 Mean sum of square and $p$ value results for the ratio of hysterectomy to ablation regressed on the minimum/maximum score in each SA3 area, SA3 SEIFA classification and the score-decile interaction.

\begin{tabular}{|c|c|c|c|c|c|}
\hline \multicolumn{3}{|l|}{ Minimun SEIFA score for SA3 } & \multicolumn{3}{|l|}{ Maximum SEIFA score for SA3 } \\
\hline & Mean SS & P value & & Mean SS & P value \\
\hline SEIFA score (min) & 0.019 & 0.791 & SEIFA score (max) & 0.875 & 0.082 \\
\hline SEIFA decile & 0.701 & 0.007 & SEIFA decile & 0.413 & 0.171 \\
\hline decile.score interaction & 0.386 & 0.177 & decile.score interaction & 0.202 & 0.701 \\
\hline
\end{tabular}


analysis in Table 2 shows that the SEIFA score played a small role in explaining the decreasing trend in the ratio (minimum: $p=0.791$; maximum: $p=0.082$ ). The significant contribution to the decreasing trend in the ratio was by the broad area SEIFA decile, especially for the minimum SEIFA area score $(p=0.007)$. For the maximum SEIFA score, the area SEIFA decile was not significant. This is not entirely surprising as the classification of the area SEIFA decile is generally determined by the average SEIFA in a specific SA3 area, thus little variation among the maximum SEIFA scores is expected. The relationship between the SEIFA scores and the ratio of hysterectomy to ablation holds for each SEIFA decile, indicated by the non-significant interaction effects from the regression (Table $\mathbf{2}$ ).

This study found that the surgical procedure with the greatest economic impact - hysterectomy - was more commonly performed than ablation, despite ablation being the recommended primary procedure and imposing the least cost, shorter recovery time, and lowest risk of complications. However, the preference for hysterectomy became significantly less with increasing socioeconomic status of women. While individual SEIFA scores had little effect on choice of procedure, the relationship held for all SA3 areas. This finding may indicate that direct financial costs are less of a consideration when it comes to the choice of the procedures. Indeed, the relationship may indicate an effect of opportunity cost: that women in higher socioeconomic areas are more likely to have employment opportunities that make it undesirable to take significant lengths of time away from work. This study found no evidence that surgeons were more likely to respond to a financial incentive and perform the more expensive procedure. If anything, the effect was stronger from patient preference. The obvious limitation to the study was that it was impossible from the surgical dataset to determine the parity (number of children) for the women involved. However, as the national recommendation is that endometrial ablation is only performed where the family is complete - indeed, pregnancy after ablation is almost impossible - it seems safe to assume that future fertility concerns did not affect decision-making either by the patient or the surgeon.

There are few studies published to date that have addressed decision-making in major elective medical treatments such as surgery. A national survey of general surgeons in the United States addressed the association between 'surgeon characteristics' and the tendency to recommend surgery [20]. Using a web-based survey that presented a number of hypothetical clinical scenarios with clinical equipoise (where no treatment is clearly superior to another) regarding the decision to operate. No association was found between a 'tendency to operate' and financial incentives, or indeed concerns about medical negligence claims.

Because endometrial ablation is relatively a newer surgical technique, whereas hysterectomy has been a part of established surgical practice for many years, it may be possible that this played a role in decision-making. A study from Australia examined 'socioeconomic lags' in the diffusion of higher technology health care by focusing on the diffusion of coronary procedures - either the major operation of heart bypass surgery, or the less-invasive technique of angiography - in patients with heart disease [25]. The study identified socioeconomic lags in diffusion with rates peaking earlier in higher SES patients, such that the inequality patterns were consistent with the inverse equity hypothesis. The authors concluded that there was evidence for a lag in diffusion of health technology across socio-economic groups, meaning that it is essential to consider trends over time when examining the equity impact of health technologies.

Study of procedural variation and surgical decision making is important, as pointed out by the ACSQHC:

"Not all variation in medical practice is warranted. It may reflect structural factors that mean some people have less access to health care compared to others. It may mean that factors other than patients' need or preferences are driving treatment decisions, particularly for discretionary interventions... Variation in medical practice may also mean that scarce health resources are not being put to best use. As countries face increasing pressure on health budgets, there is growing international interest in addressing unwarranted variation in medical practice, as such variation affects equity of access to appropriate services, the health outcomes of populations, and efficient use of resources."14

Australian governments have difficulty in anticipating expenditure on surgery and surgical procedures because they are demand driven [10]. For each MBS payment made surgery there is usually an associated payment for an anaesthetist and in many cases a payment to a surgical assistant as well. The majority of surgical procedures generate associated payments for pathology services and, through the Pharmaceutical Benefits Scheme (PBS), payments for medications such as antibiotics and analgesics. Surgery may also contribute to payments for treatment of operative complications. More broadly, when patients undergo surgical procedures, there is reduced productivity both for the patient and for carers.

\section{Conclusion}

The clinical variation in incidence rates of procedures for $\mathrm{HMB}$ have offered the opportunity to study trade-offs in choice of procedure: between a slightly less-effective but less expensive procedure (ablation), and a more highly-effective procedure with much greater economic impact (hysterectomy). We made the finding that patients in higher socio-economic groups were more likely to choose the former procedure. It seems they have exercised consumerist behaviour and considered non-monetary considerations such as recovery time. This is the opposite of the findings for long-term drug therapy in chronic conditions. The reasons for this finding deserve further study.

\section{Disclosures}

$\mathrm{SJR}$ is a member of the Ministerial Advisory Committee on out-ofpocket costs, and is a paid member of the Australian Government MBS Review.

${ }^{14} \mathrm{https}: / / w w w . s a f e t y a n d q u a l i t y . g o v . a u / a t l a s / a t l a s-2017 /$ 


\section{References}

1 Dieleman JL, Campbell M, Chapin A, Eldrenkamp E, Fan VY, et al. (2017) Future and potential spending on health 2015-40: development assistance for health, and government, prepaid private, and out-ofpocket health spending in 184 countries. Lancet 10083: 2005-2030.

2 Bartlett C, Butler S, Rogan C (2016) Australia's healthcare system: An opportunity for economic growth. Price Waterhouse Coopers, pp: 1-23.

3 Fuchs VR (2018) Health economics and policy. New Jersey; World scientific.

4 Shafrin J (2010) Operating on commission: analysing how physician financial incentives affect surgery rates. Health Econ 19: 562-580.

5 Labelle R, Stoddart G, Rice T (1994) A re-examination of the meaning and importance of supplier-induced demand. J Health Econ 13: 347-368.

6 Ryan M (1994) Agency in health care: lessons for economists from sociologists. The American Journal of Economics and Sociology 53: 207-217.

7 Bickerdyke I, Dolamore R, Monday I, Preston R (2002) SupplierInduced Demand for Medical Services, Productivity Commission Staff Working Paper, Canberra, November.

8 Fuchs VR (2011) The doctor's dilemma- what is "appropriate" care? N Engl J Med 365: 585-587.

9 Reschovsky JD, Kemper P (1990) Do HMOs make a difference? Introduction. Inquiry 36: 374-377.

10 Boxall A (2011-2012) What are we doing to ensure the sustainability of the health system? Research paper no. 4, 2011-2012. Parliamentary Library.

11 Davis DA, Taylor-Vaisey A (1997) Translating guidelines into practice: a systematic review of theoretical concepts, practical experience and research evidence in the adoption of clinical practice guidance. Can Med Assoc J 157: 408-416.

12 Buchan H, Sewell JR, Sweet M (2004) Translating evidence into practice. Med J Aust 180: s43.

13 Kennedy PJ, Leathly CM, Hughes CF (2010) Clinical practice variation. Med J Aust 193: 597-599.
14 Wilmshurst L (2015) What Really Drives Medical Treatment Decisions? Stanford Business Insights.

15 Wood SF, Podrasky J, McMonagle MA, Raveendran J, Bysshe T, et al. (2017) Influence of pharmaceutical marketing on Medicare prescriptions in the District of Columbia. PLoS ONE 12: e0186060.

16 Salkeld G, Solomon MJ (2003) An economic perspective on evidencebased patient choice in surgery. Aust N Z J Surg 73: 427-430.

17 Wilson NP, Wilson FP, Neuman M, Epstein A, Bell R, et al. (2013) Determinants of surgical decision making: a national survey. Am J Surgery 206: 970-978.

18 Plotzke MR, Courtemanche C (2011) Does procedure profitability impact whether an outpatient surgery is performed at an ambulatory surgery center or hospital? Health Econ 20: 817-830.

19 Cole JA, Norman H, Weatherby LB, Walker AM (2006) Drug copayment and adherence in chronic heart failure: Effect on cost and outcomes. Pharmacotherapy 26: 1157-1164.

20 Goldman DP, Joyce GF, Zheng Y (2007) Prescription drug cost sharing: Associations with medication and medical utilization and spending and health. JAMA 298: 61-69.

21 Tseng C, Waitzfelder BE, Tierney EF, Gerzoff RB, Marrero DG, et al. (2010) Patients' willingness to discuss trade-offs to lower their outof-pocket drug costs. Arch Intern Med 170: 1502-1504.

22 El-Hemaidi I, Gharaibeh A, Shehata H (2007) Menorrhagia and bleeding disorders. Curr Opin Obstet Gynaecol 19: 513-520.

23 Frick KD, Clark MA, Steinwachs DM, Langerberg P, Stovall D, et al. (2009) Financial and quality-of-life burden of dysfunctional uterine bleeding among women agreeing to obtain surgical treatment. Women Health Issues 19: 70-78.

24 Miller JD, Lenhart GM, Bonafede MM, Basinski CM, Lukes AS, et al. (2015) Cost effectiveness of endometrial ablation with the NovaSure system versus other global ablation modalities and hysterectomy for treatment of abnormal uterine bleeding: US commercial and Medicaid payer perspectives. Int J Women's Health 7: 59-73.

25 Bonafede MM, Cai Q, Miller JD, Pohlman SK, Troeger KA (2017) Economic and clinical outcomes among women with abnormal uterine bleeding treated with inpatient or outpatient hysterectomy versus endometrial ablation. J Minim Invasive Surg 24: s132. 